\title{
Fibre size and content of the anterior tibial nerve of the foot
}

\author{
MICHAEL SWALLOW ${ }^{1}$ \\ From the Institute of Neurology, National Hospital, Queen Square, London
}

In 1935, Greenfield and Carmichael made a quantitative study of the fibre content of the anterior tibial nerve of the foot in patients suffering from subacute combined degeneration of the spinal cord and from alcoholic polyneuritis. Some nerves from healthy subjects were also examined. Subsequently it was suggested by Blackwood (1952) that the anterior tibial nerve provided 'A readily accessible sensory nerve ... from which we can estimate exactly the amount of loss of myelinated fibres'. However, Garven, Gairns, and Smith (1962) have questioned the reliability of the anterior tibial nerve for quantitative study, and have drawn attention to the variability of its fibre content in normal people. The present work was designed to investigate these conflicting views and to demonstrate the extent and nature of such variability.

\section{MATERIAL AND METHODS}

Specimens of the terminal branch of the anterior tibial nerve were taken at necropsy from subjects without evidence of peripheral nerve disease. In 15 patients who died in hospital, detailed clinical records were available and in no case was there any clinical evidence of peripheral nerve disease. Material in the other eight cases was obtained from coroners' courts where the necropsies were performed by the coroners' pathologists; in most of these cases, patients had died from suicide, traffic accidents, or suddenly in their homes, and while few clinical details were available, there was no reason to suppose that disease of the peripheral nerves was present. Nerves from both feet were taken in two patients; in all, 25 nerves were obtained from 23 subjects. The age, sex, and cause of death in each case are shown in Table I.

The anterior tibial nerve was dissected out from the dorsum of the foot, and about $1 \mathrm{~cm}$. removed with a sharp razor blade. The specimen was then gently stretched over a small piece of card to which the ends were made to adhere by light pressure. Mounting under slight tension ensured that the nerve remained straight during the subsequent preparation. After mounting on cards, the specimens were placed immediately into Flemming's solution at room temperature for 24 to 48 hours. They were then dehydrated in alcohol and embedded in paraffin. Sections

${ }^{1}$ Present address: Royal Victoria Hospital, Grosvenor Road, Belfast, 12.
TABLE I

DETAILS OF SUBJECTS STUDIED

\begin{tabular}{|c|c|c|c|c|}
\hline $\begin{array}{l}\text { Case } \\
\text { No. }\end{array}$ & Age & Sex & Source ${ }^{1}$ & Diagnosis \\
\hline 1 & 76 & $\mathbf{M}$ & $\mathbf{H}$ & $\begin{array}{l}\text { Coronary thrombosis, pulmonary } \\
\text { embolus }\end{array}$ \\
\hline 2 & 62 & $\mathbf{F}$ & $\mathbf{H}$ & Crohn's disease \\
\hline 3 & 64 & $\mathbf{F}$ & $\mathbf{H}$ & Acute pyelonephritis \\
\hline 4 & 22 & $\mathbf{M}$ & C & Carbon monoxide poisoning \\
\hline 5 & 17 & $\mathbf{M}$ & C & Road traffic accident \\
\hline 6 & 46 & $\mathbf{M}$ & C & Coronary thrombosis \\
\hline 7 & 65 & $\mathbf{F}$ & C & Road traffic accident \\
\hline 8 & 22 & $\mathbf{F}$ & $\mathrm{C}$ & Carbon monoxide poisoning \\
\hline 9 & 55 & $\mathbf{M}$ & $\mathbf{C}$ & Coronary thrombosis \\
\hline 10 & 33 & $\mathbf{M}$ & $\mathbf{C}$ & Barbiturate poisoning \\
\hline 11 & 34 & $\mathbf{M}$ & $\mathbf{H}$ & Subacute bacterial endocarditis \\
\hline 12 & 55 & $\mathbf{M}$ & C & Coronary thrombosis \\
\hline 13 & 68 & $\mathbf{F}$ & $\mathbf{H}$ & Intracranial tumour (meningioma) \\
\hline 14 & 39 & $\mathbf{F}$ & $\mathbf{H}$ & Intracranial tumour (glioma) \\
\hline 15 & 15 & $\mathbf{F}$ & $\mathbf{H}$ & Hydrocephalus, aqueduct stenosis \\
\hline 16 & 47 & $\mathbf{M}$ & $\mathbf{H}$ & Intracranial tumour (glioma) \\
\hline 17 & 57 & $\mathbf{M}$ & $\mathbf{H}$ & Intracranial tumour (glioma) \\
\hline 18 & 63 & $\mathbf{M}$ & C & Carbon monoxide poisoning \\
\hline 19 & 71 & $\mathbf{M}$ & C & Myocardial failure \\
\hline 20 & 55 & $\mathbf{M}$ & $\mathbf{H}$ & Subarachnoid haemorrhage \\
\hline 21 & 28 & $\mathbf{M}$ & $\mathbf{H}$ & Road traffic accident \\
\hline 22 & 62 & $\mathbf{F}$ & $\mathbf{H}$ & Cerebral haemorrhage \\
\hline 23 & 62 & $\mathbf{M}$ & $\mathbf{H}$ & Cerebral haemorrhage \\
\hline
\end{tabular}

'Source: $\mathbf{H}=$ hospital; $\mathbf{C}=$ coroner's court.

$5 \mu$ thick were cut and stained with Kultschitzky's haematoxylin as described by Gutmann and Sanders (1943). They were then dehydrated, cleared, and mounted.

This method was found to give good contrast with minimal distortion of nerve bundles or fibres. The myelin sheaths stain black, and counterstaining with light green was used in some cases to show the connective tissue and perineurial sheaths more clearly. In some of the larger bundles, however, the staining was uneven, that part of the bundle nearest to the surface of the nerve showing better and more even staining than the fibres deeper in the bundle; this was probably due to poor penetration of the fixative. More even staining was obtained by cutting the nerve transversely after it had been in the fixative for a few hours, thus allowing the fixative to penetrate through the cut ends.

The amount of shrinkage inherent in this method of preparation has been discussed by several authors. Shrinkage may occur at the time of removal of the specimen, during fixation, or during dehydration and embedding. Careful dissection and rapid transfer to the fixative probably eliminate any shrinkage during removal 
from the body. Duncan (1934), Rexed (1944), and Sanders (1948) all state that there is no significant change in diameter during fixation in osmium-containing fluids. It is likely, however, that some shrinkage occurs during dehydration and embedding; this was estimated by Sanders (1948), and accepted by Fernand and Young (1951) as causing a 6 to $8 \%$ reduction in fibre diameter. Hursh (1939) demonstrated $10 \%$ shrinkage of osmiumfixed teased fibres during dehydration. The shrinkage affects axons and myelin sheaths to an equal extent, and fibres of all diameters are affected equally, thus preserving their relationship to one another with regard to size.

All the sections were photographed at a magnification of $\times 250$ on a Zeiss Ultraphot II camera-microscope, using $9 \times 12 \mathrm{~cm}$. Ilford panchromatic plates. Enlargements were made on to bromide paper and two sets of prints were produced: $(a)$ all sections were printed at a magnification of $\times 500$ for estimating total fibre counts and $(b)$ selected nerves were enlarged to $\times 1,000$ for measurement of fibre diameter. During the process of enlarging, magnification was checked regularly using a plate on which a micrometer scale had been photographed. Myelinated fibres with an external diameter of $2 \mu$ or less were not always clearly delineated in enlarged photographs; it was therefore essential to check the photographic appearances against the original slides, using high magnification and an oil-immersion lens when necessary.

Total fibre counts for each nerve were made, using a sharpened metal pointer which penetrated the photograph and made contact with a metal base-plate, thus registering the count on an automatic counter. When individual fascicles were circular in shape, the area of each was calculated from the diameter measured to the inner border of the perineurial sheath. When fascicles were oval in shape, the mean of two diameters at right angles was taken, any irregularities being checked with a planimeter. Fibre density was calculated as the number of fibres per sq. $\mathrm{mm}$. of intraperineurial area.

For the construction of histograms of fibre size, the external diameter of the myelin sheath was measured from $\times 1,000$ photographs by means of a perspex cursor and mechanical counter which allowed automatic registration of fibre diameter, as described by Espir and Harding (1961). In the preparation of the histograms, fibres with an external diameter of $2 \mu$ or less were grouped together and larger fibres were subdivided into $1 \mu$ groups.

\section{RESULTS}

The anatomy of the termination of the anterior tibial nerve and the site of section are shown in Figure 1. The nerve becomes superficial between the shafts of the first two metatarsal bones and sometimes emerges from the deep fascia already split into its two digital branches. There is usually a communication from the medial branch of the musculocutaneous nerve; in the present study the anterior tibial nerve was removed proximal to the point at which it was joined by this communicating branch,

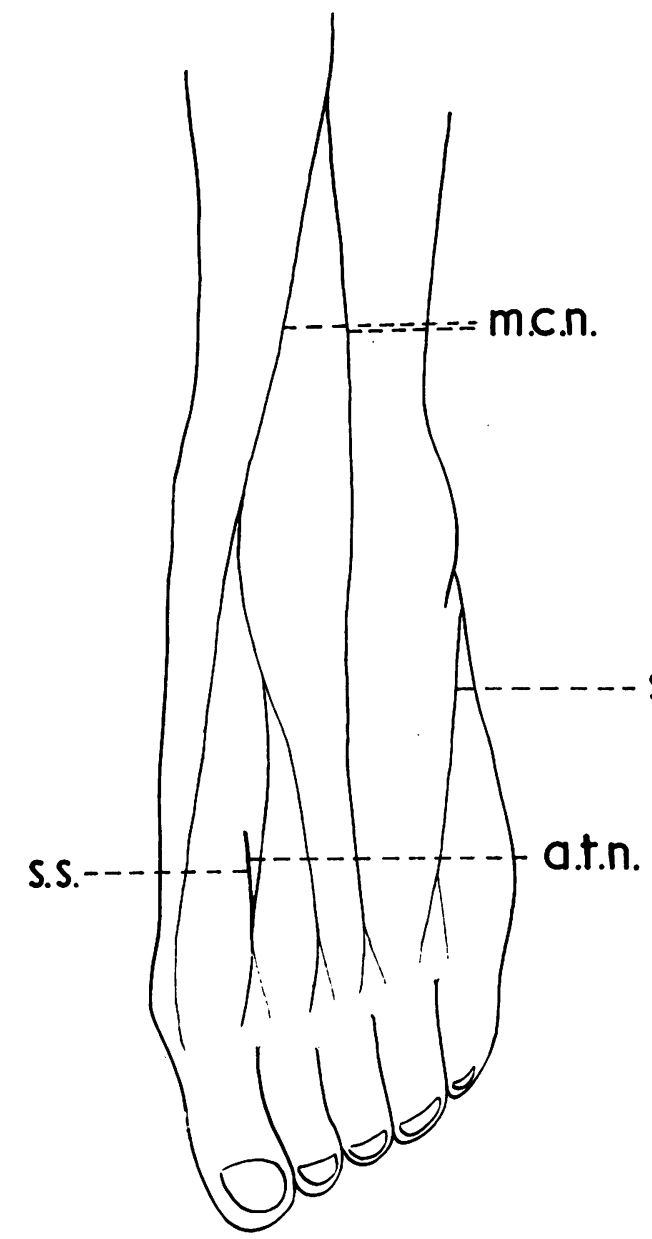

FIG. 1. Superficial nerves of the dorsum of the foot. m.c.n. = musculo-cutaneous nerve

s.n. = sural nerve

a.t.n. = anterior tibial nerve

s.s. $\quad=$ site of section

and some of the variation which was found in the size of nerves from different subjects may be due to? the fact that when the communicating branch was large, the main trunk of the anterior tibial nerve was usually small.

VARIATIONS IN THE SIZE AND NUMBER OF FASCICLES응 In most cases the diameter of individual fascicles was $>$ between $0.15 \mathrm{~mm}$. and $1.0 \mathrm{~mm}$. However, fascicles were seen with diameters in the region of $50 \mu$ and $\tilde{O}$ containing only a few nerve fibres. For such small ${ }^{\circ}$ fascicles estimates of fibre density have little mean- $N$ ing, and those with a diameter of less than $100 \mu \mathrm{N}$ have therefore been excluded from calculations of 


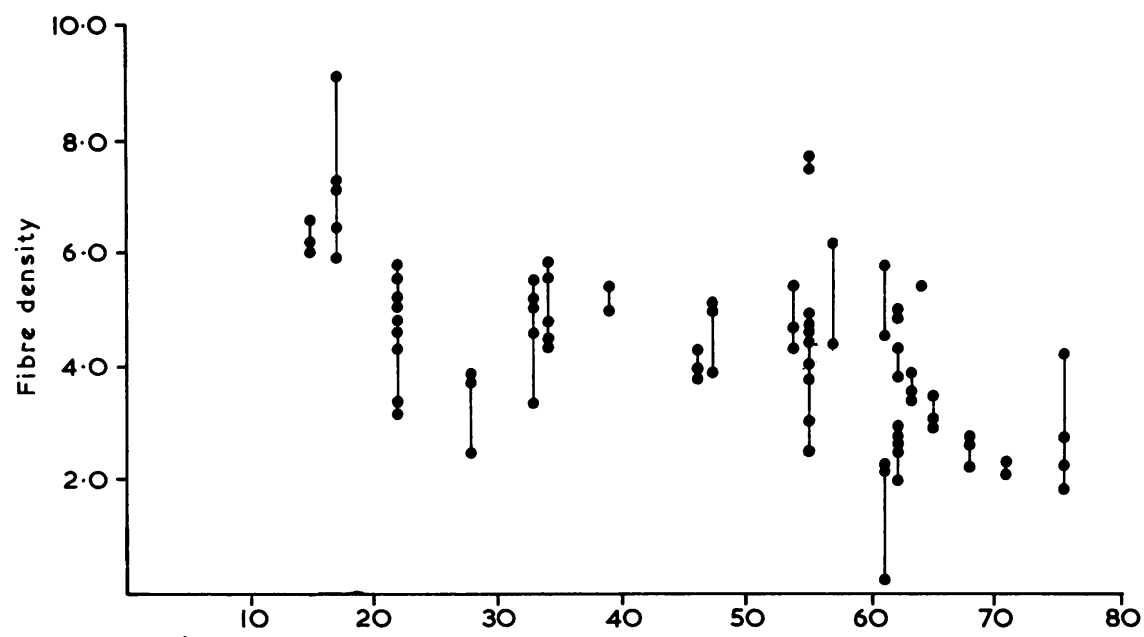

FIG. 2 Fibre

density (thousand

fibres per sq. $\mathrm{mm}$.)

of individual

fascicles plotted

against the subject's

age. Values for

different fascicles

of the same nerve

are joined by a line.

Age in years

fascicular area and fibre density. In the 25 nerves examined, there were in all 105 fascicles, of which 22 had a diameter of less than $100 \mu$. The distribution of the remaining 83 fascicles among the 25 nerves is shown in Fig. 2, and it can be seen that there was no correlation between the number of fascicles and the subject's age. Furthermore, there was no correlation between either the diameter of individual fascicles or the mean fascicular diameter and the subject's age.

From the sum of the cross-sectional areas of individual fascicles the total fascicular area was calculated for each subject. This varied from 0.126 sq. $\mathrm{mm}$. to $0.515 \mathrm{sq}$. $\mathrm{mm}$. in different subjects (Table II), with a mean of 0.273 sq. mm., and showed no correlation with age.

These results indicate that age has no effect on either the number or size of the constituent fascicles of the anterior tibial nerve, and that both may vary in a random fashion within wide limits.

VARIATION IN TOTAL FIBRE CONTENT The total number of myelinated fibres in each nerve is shown in Table II. In Fig. 3 the total fibre content is plotted against the subject's age. It can be seen that the lowest count (325 fibres) occurred in a 68-year-old female subject and the highest (2,539 fibres) in a 17-year-old male. These results indicate that the total fibre content of the anterior tibial nerve tends to decrease in older subjects, although there is also considerable random variation.

In order to test the significance of the inverse relationship between total fibre count and age, the findings in the 25 nerves were ranked according to fibre count and age, and the differences in ranking compared by calculating Spearman's rank correla-
TABLE II

TOTAL FASCICULAR AREA FOR EACH SUBJECT

\begin{tabular}{|c|c|c|c|c|}
\hline $\begin{array}{l}\text { Case } \\
\text { No. }\end{array}$ & $\begin{array}{l}\text { Total } \\
\text { Fibres }\end{array}$ & $\begin{array}{l}\text { Fibre Content } \\
\text { of Small } \\
\text { Fascicles } \\
(\text { diam. }<100 \mu)\end{array}$ & $\begin{array}{l}\text { Total } \\
\text { Fascicular } \\
\text { Area }^{1} \\
\text { (sq. mm.) }\end{array}$ & $\begin{array}{l}\text { Mean Fibre } \\
\text { Density } 1 \\
\text { (thousand fibres } \\
\text { per sq. mm.) }\end{array}$ \\
\hline 1 & 462 & - & $0 \cdot 194$ & $2 \cdot 38$ \\
\hline 2 & 869 & 25 & 0.340 & 2.48 \\
\hline 3 & 672 & - & $0 \cdot 126$ & $5 \cdot 35$ \\
\hline 4 & 1,592 & - & 0.305 & $5 \cdot 22$ \\
\hline 5 & 2,539 & 10 & 0.382 & 6.64 \\
\hline 6 & 1,220 & - & 0.306 & 3.99 \\
\hline 7 & 516 & - & $0 \cdot 161$ & $3 \cdot 21$ \\
\hline 8 & 1,522 & 36 & 0.368 & 4.04 \\
\hline 9 & 1,066 & 14 & $0 \cdot 200$ & 5.25 \\
\hline 10 & 742 & - & $0 \cdot 150$ & 4.94 \\
\hline 11 & 2,307 & 23 & 0.473 & 4.84 \\
\hline 12 & 2,131 & 32 & 0.515 & 4.07 \\
\hline 13 & 325 & - & $0 \cdot 127$ & 2.56 \\
\hline 14 & 1,979 & - & $0 \cdot 154$ & 5.04 \\
\hline 15 & 2,033 & - & 0.310 & $6 \cdot 27$ \\
\hline 16 & 809 & 52 & $0 \cdot 162$ & 4.67 \\
\hline 17 & 1,225 & 8 & 0.258 & 4.72 \\
\hline 18 & 1,121 & - & 0.308 & 3.64 \\
\hline 19 & 530 & - & 0.241 & $2 \cdot 19$ \\
\hline 20 & 1,374 & - & 0.182 & 7.56 \\
\hline 21 & 1,451 & 89 & 0.414 & 3.29 \\
\hline 22R & 1,163 & - & 0.242 & 4.81 \\
\hline $22 \mathrm{~L}$ & 1,466 & 9 & 0.370 & 3.94 \\
\hline $23 R$ & 1,485 & 28 & 0.292 & 4.99 \\
\hline $23 \mathrm{~L}$ & 469 & - & 0.259 & 1.81 \\
\hline
\end{tabular}

${ }^{1}$ Fascicles less than $100 \mu$ in diameter omitted.

tion coefficient (R). This was found to be 0.74 : Student's t test shows that this is significant at the 0.001 probability level.

VARIATION IN FIBRE DENSITY Fibre density may be expressed as the number of fibres per square millimetre of intra-perineurial area. This has been calculated for 83 individual fascicles in the 25 nerves, 


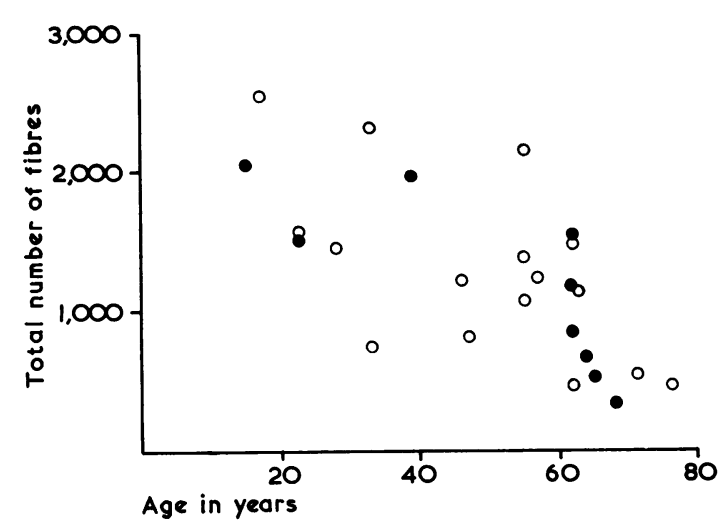

FIG. 3. Total fibre content of anterior tibial nerve plotted against subject's age. (Open circles for male and filled circles for female subjects.)

only those fascicles with a diameter of less than $100 \mu$ being omitted. The results are shown in Figure 2. Variation in fibre density was found to occur not only between different nerves but also between one fascicle and another in the same nerve. For example, it can be seen that in the 17-year-old patient whose anterior tibial nerve contained five fascicles, fibre density varied from 5,900 to 9,088 per sq. $\mathrm{mm}$.

Mean fibre density for each of the 25 nerves has been calculated by adding together the numbers of myelinated fibres in each fascicle (fascicles of less

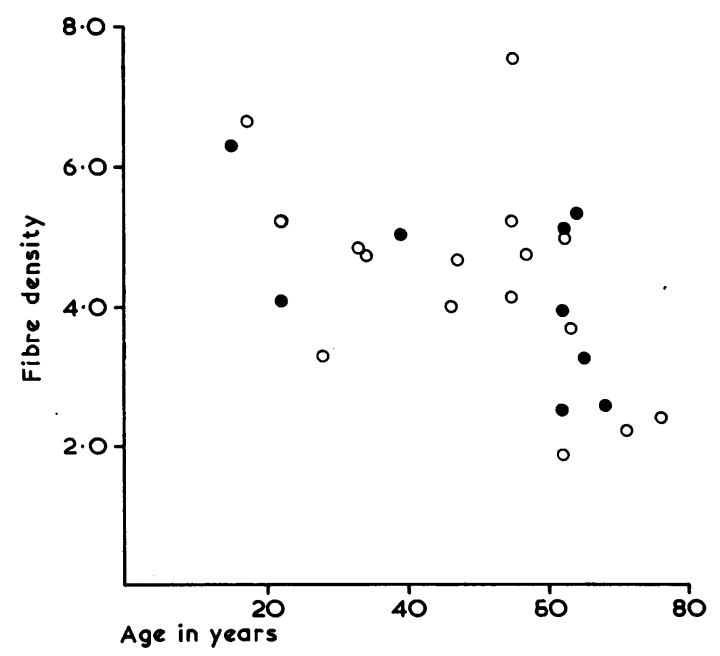

FIG. 4. Mean fibre density (thousand fibres per sq. $\mathrm{mm}$.) of anterior tibial nerve plotted against subject's age. (Open circles for male and filled circles for female subjects.) than $100 \mu$ diameter being ignored), and then $\frac{\bar{c}}{Z}$ dividing the total number of fibres in each nerve by $\underset{\mathbb{D}}{ }$ the sum of the intra-perineurial areas of individual fascicles. The results are shown in Table II. In Fig. $4 \mathrm{en}$ fibre density is plotted against the subject's age, and a general trend towards decreasing fibre density with 0 increasing age can be seen. For example, the two youngest subjects (aged 15 and 17) both showed $\stackrel{\circ}{=}$ fibre densities of over 6,000 per sq. mm., whereas in the two oldest subjects (aged 71 and 76) the fibre density was below 2,500 per sq. $\mathrm{mm}$. Using Spear- $\overrightarrow{\vec{F}}$ man's ranking test, the inverse relationship between fibre density and age for the 25 nerves examined was $\frac{}{0}$ significant at the 0.01 probability level $(R=0.57)$.

In Fig. 3 and Fig. 4, values for the total fibre count $\vec{\Phi}$ and fibre density are shown as open circles for male $\frac{\varrho}{\vartheta}$ subjects and filled circles for female. These figures do $\infty$ not suggest a systematic difference between the $\overrightarrow{0}$ sexes, and when mean values for the two groups are $\overrightarrow{ }$ calculated (Table III), it is clear that there is now substantial difference between male and female subjects.

\section{TABLE III}

\begin{tabular}{|c|c|c|c|}
\hline MEAN FIBRE COUN' & MEAN DEN & TY & \\
\hline & $\begin{array}{l}\text { Males } \\
\text { (16 nerves) }\end{array}$ & $\begin{array}{l}\text { Females } \\
\text { ( } 9 \text { nerves) }\end{array}$ & \\
\hline $\begin{array}{l}\text { Mean fibre count } \\
\text { Mean density } \\
\text { (thousand fibres per sq. mm.) }\end{array}$ & $\begin{array}{r}1,282 \\
4 \cdot 39\end{array}$ & $\begin{array}{r}1,172 \\
4 \cdot 19\end{array}$ & \\
\hline
\end{tabular}

In two subjects the anterior tibial nerve was taken from both sides for comparison. It can be seen from Table II that in case 22 the nerves from the two sides were similar; in case 23 , however, there was great 0 disparity between the right and left, one of the \% bundles from the left side containing only $10 \AA$ myelinated fibres (Fig. 5). In this subject it might be $\overrightarrow{\vec{O}}$ asked whether obliterative arterial disease could have 3 been present in the legs to account for these changes, $\supset$ as in the cases described by Garven et al. (1962). Although examination of the vasa nervorum in the biopsy specimen of case 23 provides no evidence of this process, it is still possible that atheromatous changes might have been present in the larger vessels 3 . at a higher level in the limb.

VARIATION IN FIBRE SIZE AND DISTRIBUTION Twelve of the 25 nerves were selected for the study of fibre diameter. Selection depended mainly upon the quality of the preparation, those showing the best and most uniform staining, with a minimum of dis- $\sim$ tortion, being used for the construction of histo- $N$ grams. However, care was taken to include nerves from all the age groups studied. Histograms were 

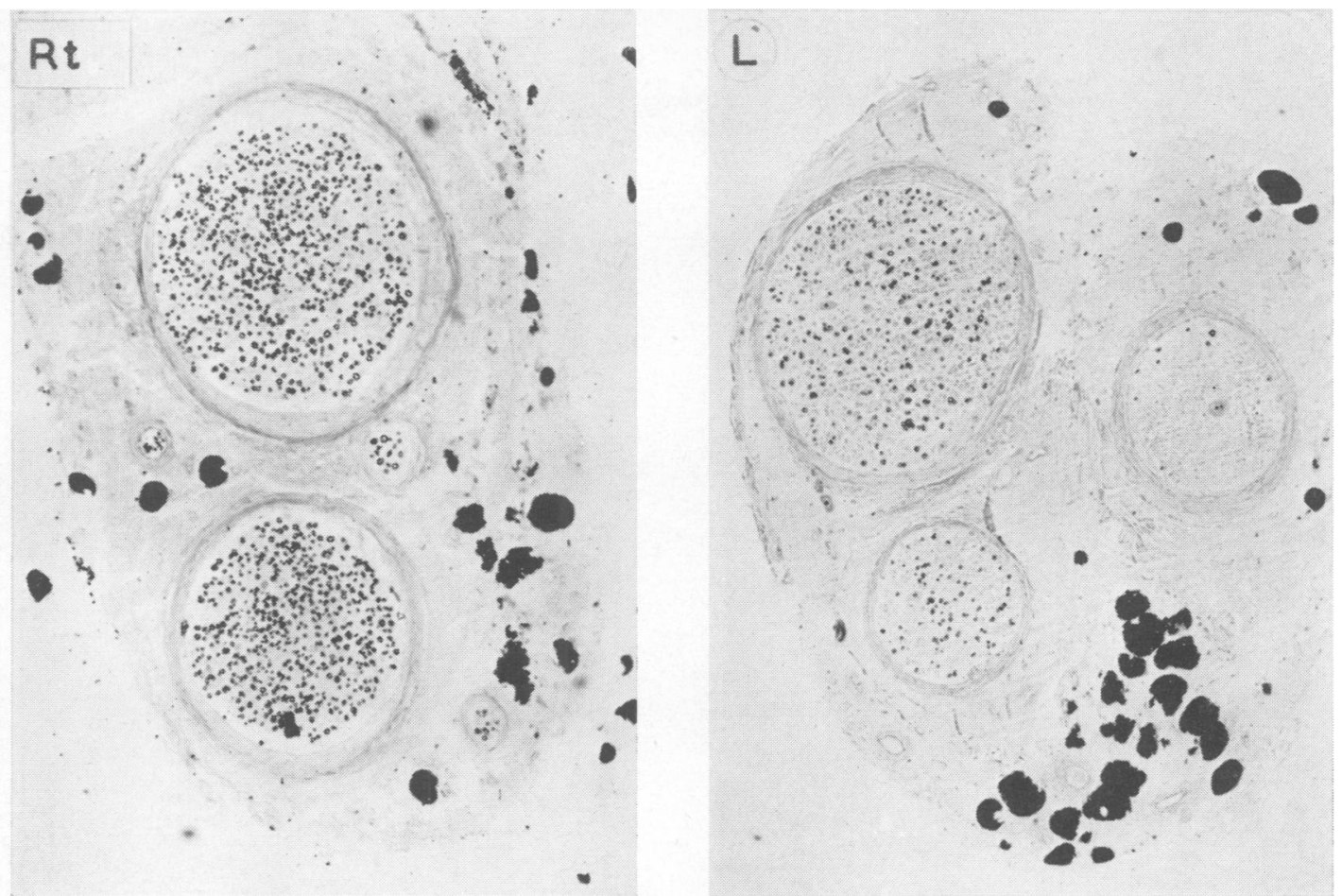

FIG. 5. Case 23, male, aged 62, to show difference in fibre content of $R$. and L. anterior tibial nerves $(\times 100)$.

plotted showing the absolute numbers of fibres from $2 \mu$ to $16 \mu$ in $1 \mu$ groups.

Figure 6a shows the nerve of a 39-year-old subject, in which there are two fascicles. Part of the nerve is shown at higher magnification in Fig. 6b. The histograms are shown in Fig. 7, and it can be seen that results for the two fascicles are similar; in both cases there is a bimodal distribution of fibre diameter with peaks at $5 \mu$ and at $10 \mu$.

Histograms were constructed from different fascicles of the same nerve in two other subjects, and were also found to be similar, although the fibre density of the fascicles varied. In these cases all the fibres were measured, but the remaining histograms were constructed from one or more of the larger fascicles in each nerve, not less than 500 fibres being measured in each case. This number was chosen in view of the observation of Rexed (1944) that 400 to 700 fibres were sufficient to give a reliable sample of fibre diameter in the case of the nerve roots.

Histograms for the 12 nerves studied are shown in Fig. 8, those obtained from subjects under the age of 50 being shown in the left hand column, and those obtained from subjects over the age of 50 being shown on the right. The histograms from the younger subjects show a bimodal distribution but in the older subjects the large fibre peak is diminished or absent. This implies that some loss of large myelinated fibres occurs with advancing age.

To examine this possibility statistically, the number of fibres with diameters of 8 to $12 \mu$ was calculated as a percentage of the total number of fibres in each histogram. The values ranged from $13 \%$ to $47 \%$ in different subjects, and when data from the 12 subjects were submitted to Spearman's ranking test, the inverse relation between age and the percentage of large fibres was significant at the 0.05 probability level $(R=0.59)$.

\section{DISCUSSION}

When the present results are compared with those of Greenfield and Carmichael (1935), it is clear that the fibre densities found in their control subjects were substantially higher than those reported in the present paper. Greenfield and Carmichael found mean densities of 6,988 to 8,602 myelinated fibres per sq. $\mathrm{mm}$. in five control subjects, with a range of 5,560 to 9,600 per sq. $\mathrm{mm}$. in individual fascicles: the ages of their five subjects were not mentioned. In the present work subjects under the age of 60 showed a mean density range of 3,293 to 7,565 


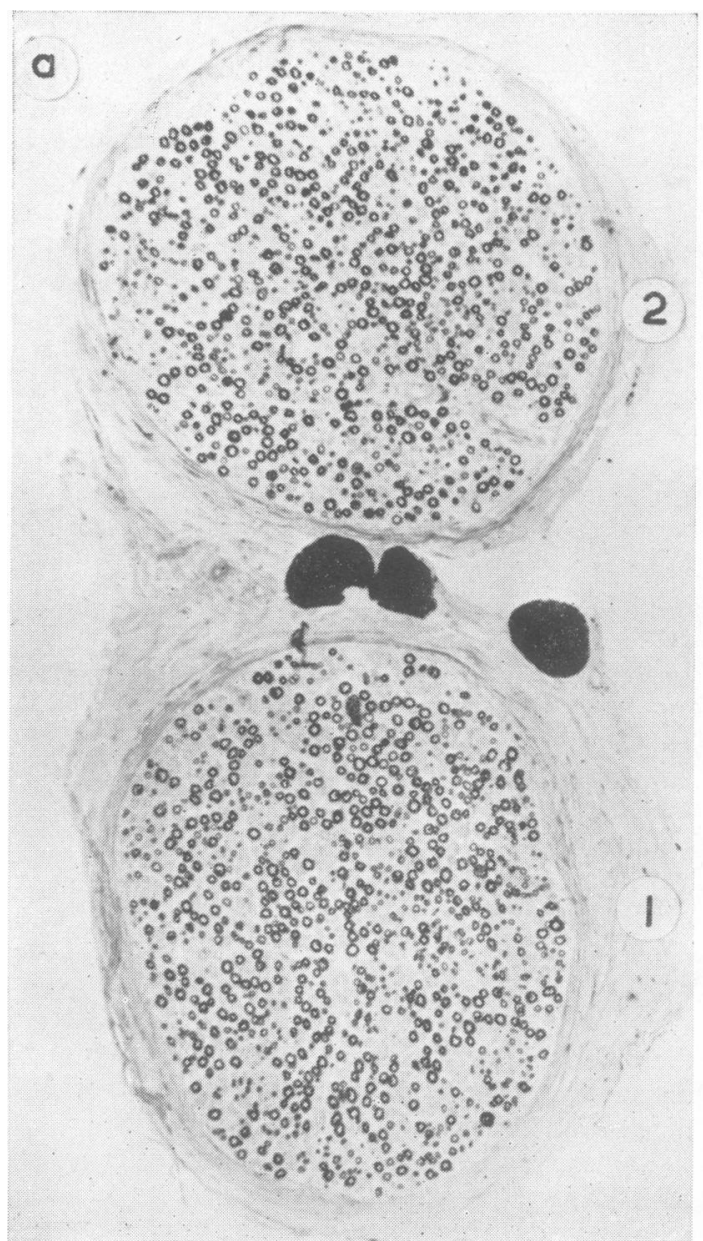

FIG. 6. Case 14, female, aged 39.

(a) Anterior tibial nerve at low magnification $(\times 100)$ to show both fascicles.

(b) Part of fascicle 2 at high magnification $(\times 300)$.

myelinated fibres per sq. $\mathrm{mm}$. in 15 subjects, with a range of 2,494 to 9,088 per sq. $\mathrm{mm}$. in individual fascicles; even lower figures were obtained for subjects over the age of 60 . In one elderly control subject studied by Garven et al. (1962), mean fibre density for the anterior tibial nerve was 11,713 per sq. mm., a higher figure than any obtained by Greenfield and Carmichael or in the present study.

These differences do not seem to be related to the exact site from which specimens of nerve were taken, as Garven et al. (1962) took their specimen from the anterior tibial nerve slightly higher than in the present work, whereas Greenfield and Carmichael took the digital branches of the nerve at a lower level. However, methods of fixation and staining differed in the three studies and may well account for the differences observed. Greenfield and Carmichael fixed and stained their material with $1 \%$ ? osmium tetroxide, whereas Garven et al. (1962) $\frac{0}{3}$ fixed in $12 \%$ formalin, followed by staining with $1 \%$. osmium tetroxide. As the latter authors admit, some shrinkage occurs during formalin fixation which is $₹$ avoided by the use of osmium-containing fixatives; 의 this might be expected to reduce fascicular area and $D$ thus to increase fibre density. Further shrinkage occurs during dehydration and embedding in $N$ paraffin, the extent of this depending upon the exact 5 technique used. Shrinkage at this stage may well have $N$ occurred in Greenfield and Carmichael's material, as they did not find any myelinated fibres in their 


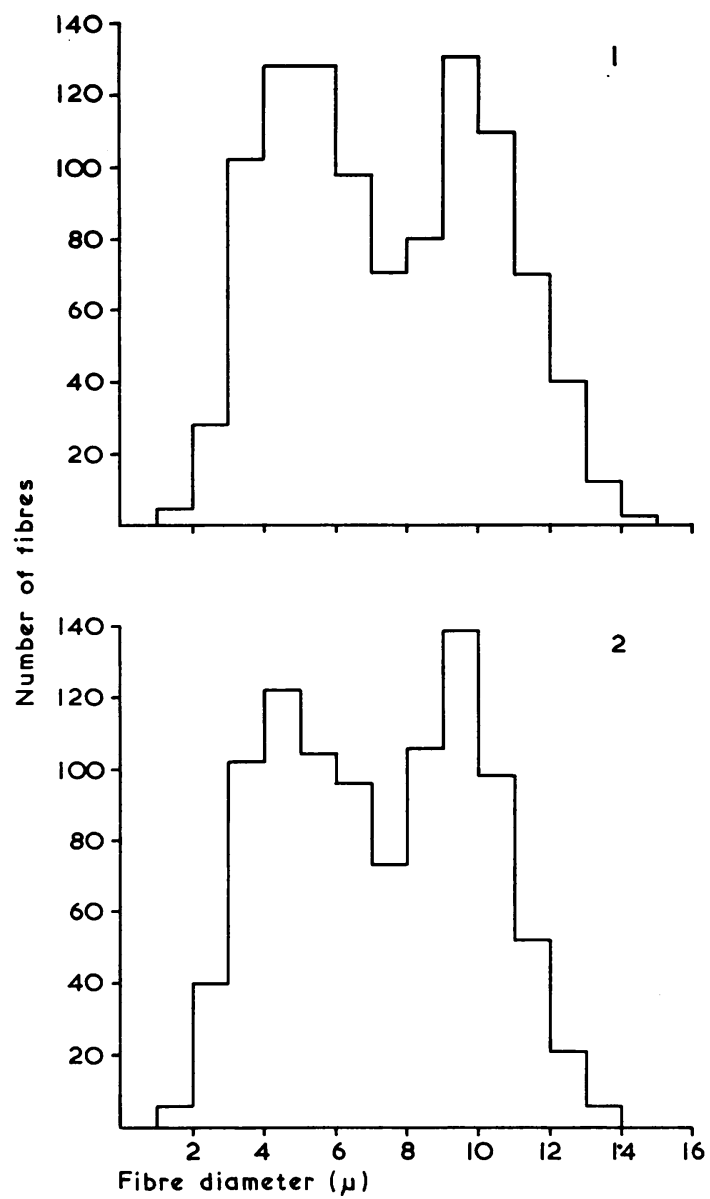

FIG. 7. Histograms of fibre diameter for the fascicles shown in Figure $6 a$.

Fascicle 1. 1,016 fibres; density 5,390 per sq. $\mathrm{mm}$.

Fascicle 2. 963 fibres; density 4,906 per sq. $\mathrm{mm}$.

control nerves with diameters greater than $10 \mu$, whereas fibres up to $14 \mu$ were found in the present work.

An alternative explanation of the low fibre densities in the present series might be that the technique used (staining with Kultschitsky's haematoxylin followed by differentiation by Pal's method) stains the smallest myelinated fibres less well than $1 \%$ osmium tetroxide. This was suggested by Rexed (1944), and it raises the possibility that fewer fibres in the 1 to $2 \mu$ range have been counted in the present work than in the two previous studies mentioned, although great care was taken to check photographs against the original slides when counting, to ensure that no fibres which had been faintly stained were omitted. However, some recent figures for fibre density in the sural and great auricular nerves have been published by Dyck, Beahrs, and Miller (1965), who used $1 \%$ osmium tetroxide staining with cryostat sections. Their figures are only slightly higher than those given in the present paper, mean densities of less than 5,000 per sq. $\mathrm{mm}$. occurring in several of their control nerves. This does not suggest that the technique used in the present study has introduced serious errors due to shrinkage or failure to stain the smallest myelinated fibres.

By their technique, Garven et al. (1962) regard a figure of the order of 10,000 to 13,000 myelinated fibres per sq. $\mathrm{mm}$. as the normal density for the sciatic nerve and its branches; they comment that the larger nerve trunks give more consistent results than the terminal sensory branches in the foot which may be subject to considerable variation. For example, one of their 'normal' digital nerves, from a young woman who had been killed in a motor accident, had a fibre density of only 2,664 per sq. $\mathrm{mm}$., whereas the corresponding figure for the sciatic nerve was 11,953 per sq. $\mathrm{mm}$.

Substantial variation in both total fibre counts and the fibre density has been seen in the present series, and it seems justifiable to conclude that pathological changes in the anterior tibial nerve and its branches can only be assessed correctly if sufficient control data are available to indicate the range of normal variation. While this variation may be less for the anterior tibial nerve than for its terminal digital branches, it is still considerable, and it is markedly increased in elderly subjects. In spite of this, the present study has shown an inverse relationship between fibre density and the subject's age which was significant at the 0.01 probability level. However, the inverse relationship between the total fibre count and the subject's age was significant at the 0.001 probability level. This suggests that there is no advantage to be gained by expressing results in the form of fibre density when the nerve concerned is small enough to allow total myelinated fibre counts to be made.

Loss of myelinated fibres with increasing age has been reported previously for the nerve roots by Corbin and Gardner (1937) and by Rexed (1944). Whether these changes are wholly due to arterial degeneration is uncertain. Changes in the vasa nervorum and in the perineurial thickness with increasing age have been described by Cottrell (1940), but other factors such as repeated minor trauma might contribute to the loss of nerve fibres. Indeed Gairns, Garven, and Smith (1960) suggest that badly fitted shoes might damage digital nerve fibres in the foot, and thus be responsible for the low fibre densities and the degenerative changes in sensory endorgans which they observed in young subjects. 

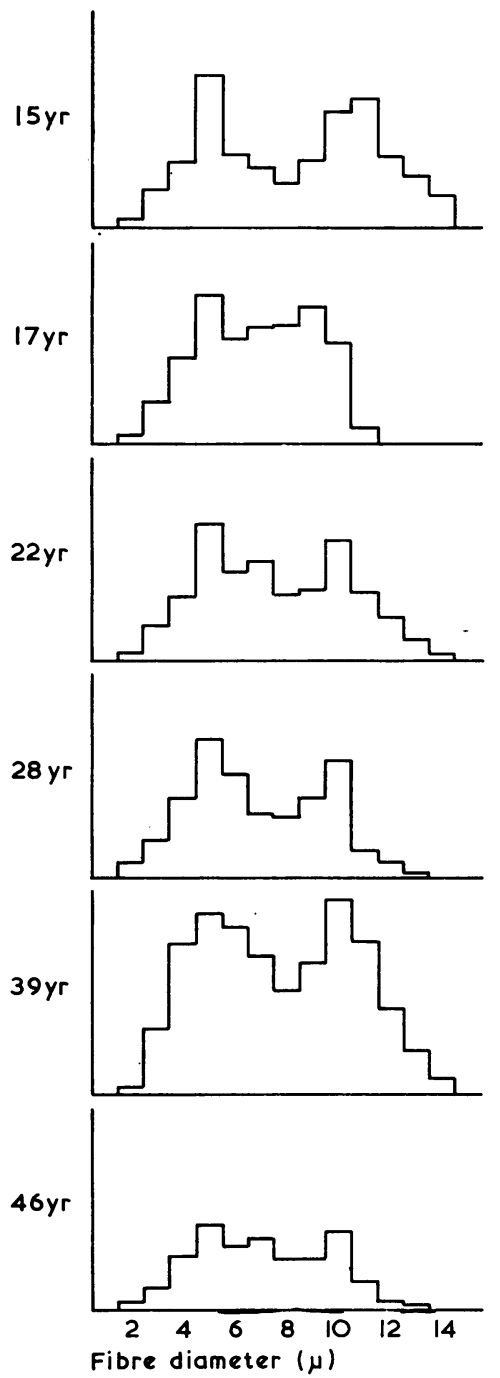
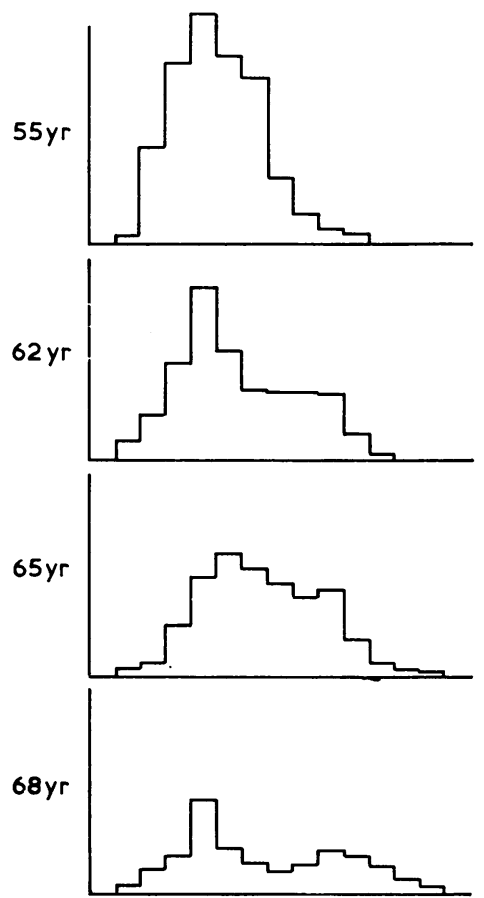

FIG. 8. Changes in pattern of fibre diameter shown by histograms from 12 subjects with ages ranging from 15 to 76 years. Age of subject shown on $L$. in each case.
Systematic observations on the blood vessels and perineurial sheaths in the present material would be of interest in this respect, and it is hoped that this will be the subject of a separate study.

In interpreting the apparently selective loss of large diameter fibres in elderly subjects, the recent observations of Lascelles and Thomas (1966) must be considered. In the sural nerves of elderly subjects Lascelles and Thomas found some fibres which had regenerated after complete degeneration, and others which showed remyelination after segmental demyelination. Both of these processes would tend to reduce the proportion of large diameter fibres seen in transverse sections, and they might be expected to contribute to the altered histograms of the elderly subjects shown in Figure 8. However, in many of these subjects the total fibre count was also markedly $\dot{0}$ reduced, from which it must be concluded that many fibres had degenerated and disappeared altogether, $\delta$ in addition to those which were capable of regeneration or remyelination.

\section{SUMMARY AND CONCLUSIONS}

The myelinated fibre content of the anterior tibial o nerve has been studied in necropsy specimens from $N$ subjects thought to be free from disease affecting the N peripheral nerves. Twenty-five nerves were obtained 
from 23 subjects whose ages ranged from 16 to 82 years. There was a highly significant fall in the total fibre count and in fibre density with increasing age.

The frequency distribution of fibres of different sizes was studied in 12 cases. A significant decrease in the proportion of large fibres was found in the older subjects.

The wide range of variation found in young as well as old subjects would seem to limit the usefulness of anterior tibial nerve biopsy as a diagnostic procedure in patients with generalized neurological disease.

This work was carried out in the University Department of Clinical Neurology, with the aid of a generous grant from the Polio Research Fund. I wish to thank Professor R. W. Gilliatt for encouragement and help.

\section{REFERENCES}

Blackwood, W. (1952). Biopsy technique in the diagnosis of peripheral neuropathies (especially hereditary and sensory neuropathy). In Proc. Ist int. Congr. Neuropath., Rome, 1925, Vol. 3, pp. 415-424.

Corbin, K. B., and Gardner, E. D. (1937). Decrease in number of myelinated fibers in human spinal roots with age. Anat. Rec., 68, 63-74.

Cottrell, L. (1940). Histologic variations with age in apparently normal peripheral nerve trunks. Arch. Neurol. Psychiat. (Chic.), 43, 1138-1150.
Duncan, D. (1934). A relation between axon diameter and myelination determined by measurement of myelinated spinal root fibers. J. comp. Neurol., 60, 437-472.

Dyck, P. J., Beahrs, O. H., and Miller, R. H. (1965). Peripheral nerves in hereditary neural atrophies: number and diameters of myelinated fibers. In Proc. 6th int. Congr. Electroenceph. clin. Neurophysiol., Vienna, pp. 673-677.

Espir, M. L. E., and Harding, D. T. C. (1961). Apparatus for measuring and counting myelinated nerve fibres. J. Neurol. Neurosurg. Psychiat., 24, 287-290.

Fernand, V. S. V., and Young, J. Z. (1951). The sizes of the nerve fibres of muscle nerves. Proc. roy. Soc. B., 139, 38-58.

Gairns, F. W., Garven, H. S. D., and Smith, G. (1960). The digital nerves and the nerve endings in progressive obliterative vascular disease of the leg. Scot. med. J., 5, 382-391.

Garven, H. S., Gairns, F. W., and Smith, G. (1962). The nerve fibre populations of the nerves of the leg in chronic occlusive arterial disease in man. Ibid., 7, 250-265.

Greenfield, J. G., and Carmichael, E. A. (1935). The peripheral nerves in cases of subacute combined degeneration of the cord. Brain, 58, 483-491.

Gutmann, E., and Sanders, F. K. (1943). Recovery of fibre numbers and diameters in the regeneration of peripheral nerves. $J$. Physiol. (Lond.), 101, 489-518.

Hursh, J. B. (1939). Conduction velocity and diameter of nerve fibers. Amer. J. Physiol., 127, 131-139.

Lascelles, R. G., and Thomas, P. K. (1966). Changes due to age in internodal length in the sural nerve in man.J. Neurol. Neurosurg. Psychiat., 29, 40-44.

Rexed, B. (1944). Contributions to the knowledge of the post-natal development of the peripheral nervous system in man. Acta phychiat. neurol. (Stockh.), suppl. 33.

Sanders, F. K. (1948). The thickness of the myelin sheaths of normal and regenerating peripheral nerve fibres. Proc. roy. Soc. B., $135,323-357$. 